\title{
A CLINICAL STUDY OF THE NUMERICAL VARIATION WITH AGE OF COMPACT NERVE ENDINGS IN THE HUMAN CONJUNCTIVA
}

\author{
P. M. RIISAGER \\ From the Department of Neurology, United Oxford Hospitals, Oxford
}

The nervous elements of the living human eye were first demonstrated by Knüsel and Vonwiller (1922) using instillations of aqueous methylene blue (without local anaesthetic) into the conjunctival sac. Nerve fibres, including 'compact' nerve endings 40 to $200 \mu$ in size, were demonstrated in the scleral conjunctiva but the technique was not used as an adjunct to clinical examination. Oppenheimer, Palmer, and Weddell (1958) adapted the technique so that it caused less discomfort to the subject. They experienced some unevenness of staining, but confirmed that many nerve fibres ended as diffuse arborizations, and that compact endings were present in appreciable numbers in man, though they were rare in the scleral conjunctiva of common experimental animals. Bullocks over 2 years old were the only other animals which showed compact terminals; they were absent in the calves. In eight normal individuals they showed that these compact endings were much more plentiful in adults than in children, some of whom had none.

Frey (1859) and his school were the first to suggest that compact nerve endings and diffuse aborizations subserved particular sensory modalities, although later Ruffini (1906) insisted that the taxonomic classification of peripheral nerve endings was arbitrary. Stimulation of individual nerve endings in the conjunctiva by Strughold and Karbe (1925) failed entirely to elicit any particular sensation.

As Oppenheimer and his colleagues pointed out, men and cattle were the only species in whom compact nerve endings could be demonstrated. Moreover, they were the only species examined where the scleral conjunctiva is normally partly exposed to the environment. If these compact nerve endings are products of regeneration and degeneration in relation to nerve fibres, their demonstration by a simple technique is obviously of interest in relation to clinical neurological procedures. The present study is concerned with the influence of age on the number of these conjunctival endings in normal individuals.
METHODS

Twelve men and 19 women between the ages of 14 and 76 , admitted for neurological investigation to the Churchill Hospital, were examined. None had symptoms or signs referable to the fifth cranial nerve, or any local abnormality of the eye or orbit.

Conjunctival staining was preceded by three applications of cocaine hydrochloride, $4 \%$ in water, at fiveminute intervals, by means of a dropper. Five minutes after the third dose, 5 drops of methylene blue (medicinal) $0.4 \%$ in $4 \%$ aqueous cocaine hydrochloride, were carefully placed in the eye to be examined and the eyelids closed. The patient was instructed to roll his eyes beneath the shut lids in order to spread the dye evenly in the conjunctival sac. More methylene blue was instilled, in like manner, at five-minute intervals until the dye had been applied five times.

The bulbar conjunctiva was examined between one and one and a half hours later. The eyelids were retracted and a gentle current of air blown over the eye for 30 seconds so that as much as possible of the reduced leuco-, form of methylene blue was oxidized to the coloured form of the dye. Failure to do this probably accounted for the uneven staining noted by Oppenheimer et al.

Compact nerve endings were counted using an ophthalmoscope with a +20 or +30 lens. Compact endings were defined as any blue dot or swelling terminating a stained nerve fibre. The total for each quadrant of the bulbar conjunctiva was noted in turn. Blue dots or swellings with no nerve fibre visible under these conditions were discounted.

Any slight discomfort which occurred up to four hours after staining was eased by application of cocaine eye drops. Each patient was warned that the eye would remain at least partly anaesthetic for up to three days, and that he must avoid touching the cornea during this time. Because of the possible danger of corneal abrasion, the investigation was not carried out on demented patients. No instance of corneal abrasion was observed.

The stained nervous elements were inspected more closely through a corneal microscope, with a lens having a focal length of $4.5 \mathrm{~cm}$., utilizing a ring of small bulbs coaxial with the microscope as a source of illumination. Photographs were taken using a ring-shaped flash tube again coaxial with the microscope and placed a little behind the objective. 


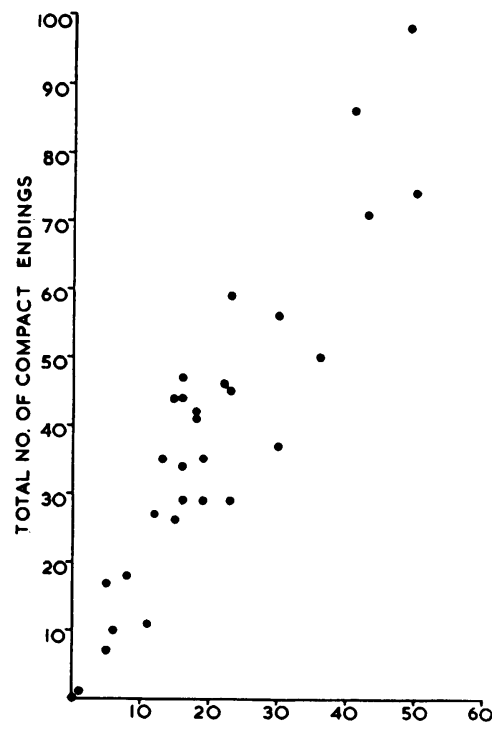

ENDINGS IN SUPERO-LATERAL QUADRANT

FIG.1. The relationship between the total number of compact conjunctival endings in the scleral conjunctiva and the total in the supero-lateral quadrant alone.

\section{RESULTS}

Conjunctival nerve fibres in all patients took up the dye. Compact terminations to nerve fibres were found in all cases except in one man of 23 years. There was no evidence of uneven staining.

Approximately half the compact endings in each case were found in the lateral, superior quadrant of the scleral conjunctiva. The very close relationship found in the present study between total count and the total in the supero-lateral quadrant is shown in Fig. 1.

The present study also demonstrates a relationship between the total number of compact nerve endings in the scleral conjunctiva and the age in normal people (Fig. 2). This correlation is highly significant (correlation coefficient, $\mathrm{r}=+\mathbf{0} \cdot 89$ ) and it seems that the number of endings increases by about 1.25 for each year of life. Thus, the best prediction of the total endings is $1.25 \times$ age less $17 \cdot 5$. This suggests that the 'compact' endings appear at about puberty. The relation between the age and the number of compact endings in the scleral conjunctiva is given in Fig. 2, where $95 \%$ confidence limits are indicated. There is no significant sex difference in the total number of endings or their distribution.

The general appearance of the stained nervous elements is indicated in Figs. 3-5.

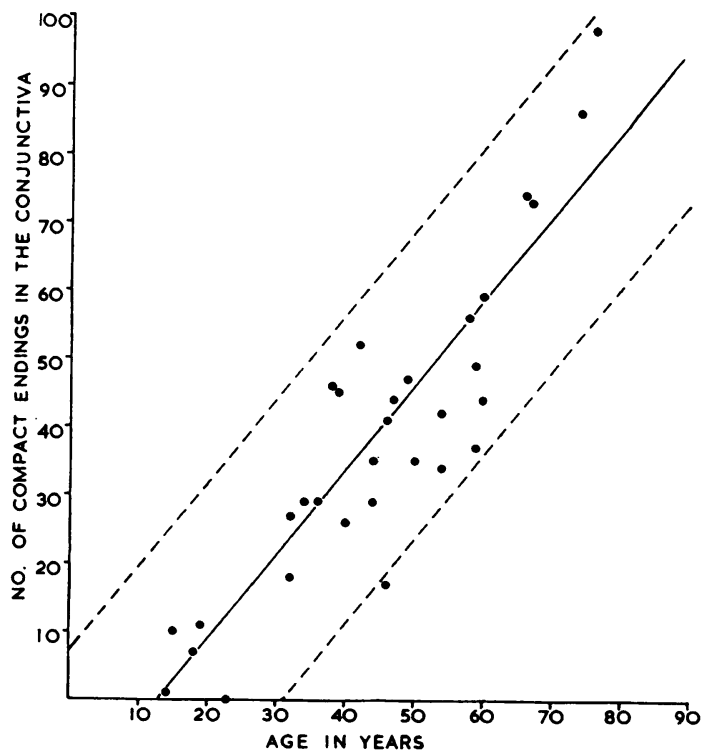

FIG. 2. The relationship between the total number of compact conjunctival nerve endings and the age of the individual $(y=1.25 \times$ age $-17 \cdot 5)$.

\section{DISCUSSION}

The incidence of compact endings in human and bovine eyes was thought to be related to exposure to the environment (Oppenheimer et al., 1958). In cattle, compact endings first appear at 2 to 3 years of age, being absent in calves. In man they are absent before puberty, and the above observations suggest that they increase directly with the age of the individual. If these compact nerve endings were specialized end organs subserving a particular sensory modality, it seems improbable that they would be absent in younger members of the species and most abundant in the elderly. It seems likely that they are associated with continued regenerative and degenerative processes, such as have been reported in other parts of the peripheral nervous system (Speidel, 1941; Zander and Weddell, 1951; Weiss, 1941).

The question remains, Do these compact endings in the scleral conjunctiva represent degenerative changes, regenerative changes, or a combination of both? Terminal swellings, of various shapes, of nerve fibre were shown by Ramón y Cajal (1928) in experimentally induced degeneration and regeneration in peripheral nerve. In particular, large 'swellings' were occasionally found to be composed of a complex nervous entanglement which he 


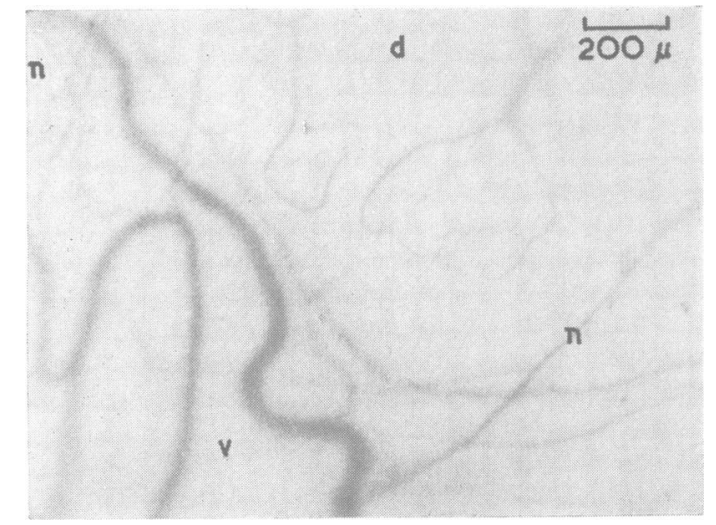

FIG. 3. Scleral conjunctiva, stained with methylene blue, in an individual aged 19. Note the fine nerve fibres, ' $n$ ', with no indication of terminal axonal swelling, which are not to be confused with fine arterial arcades, ' $d$ ', or larger vessels, ' $v$ '.

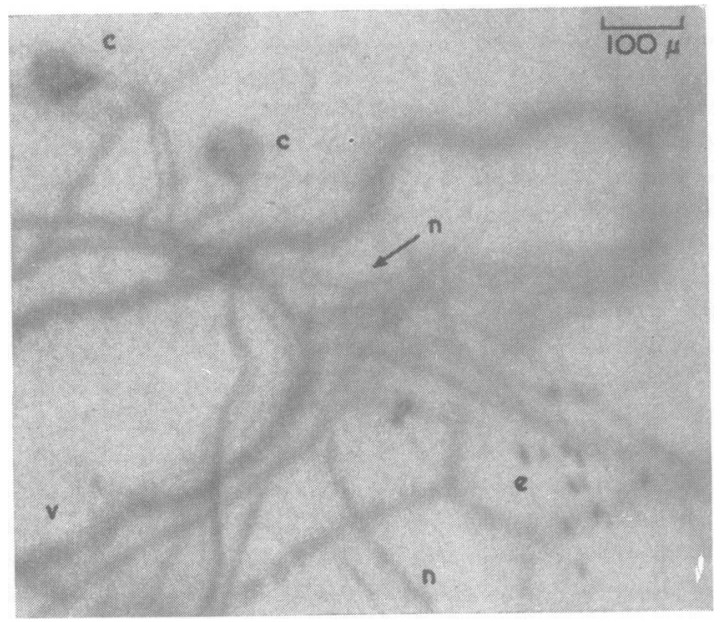

FIG. 4. Scleral conjunctiva, stained with methylene blue, in a woman of 59, showing two typical compact endings, ' $c$ ', of conjunctival nerve fibres, ' $n$ '. Some stained epithelial cells are also seen, ' $e$ '.

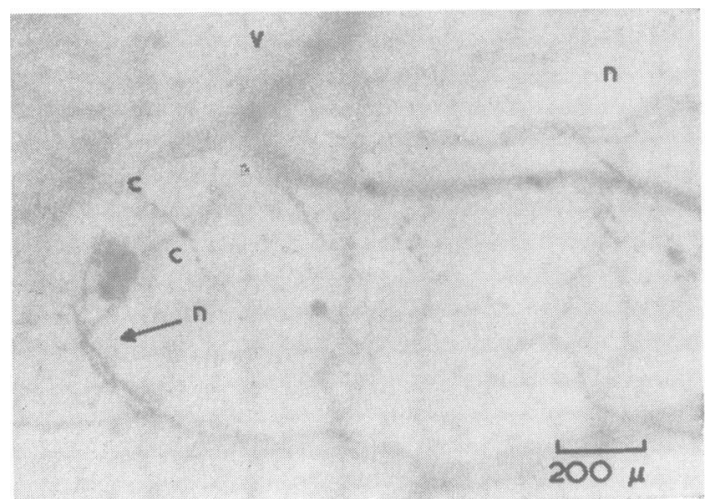

FIG. 5. Scleral conjunctiva in the same individual as in Fig. 4. There is a bundle of three nerve fibres, two of which terminate in compact endings, ' $c$ '. This overlapping occasionally results in errors in counting. described as a result of 'abortive regeneration' of a fibre. Knüsel and Vonwiller (1922) indeed described such an entanglement in the interior of a compact conjunctival ending. Ramón y Cajal also stated that regenerative axonic sprouts pursue a much more erratic course in the tissues of older animals, presumably as a result of increased tissue resistance to the sprout, due to tissue fibrosis.

This would at least partly explain the increase in these compact endings with age. Any disease process affecting the metabolism of the nerve fibre would be expected to affect the extent of degeneration and regeneration and the degree of 'abortive' regeneration. This might be expected to alter the innervation of the scleral conjunctiva. Examination of conjunctival nerve endings by the technique described is likely to be of interest in such conditions.

\section{SUMMARY}

A technique for the staining of conjunctival nerve fibres at the bed side is described.

The number of compact nerve endings occurring in the eyes of three subjects, who had no clinical involvement of the trigeminal nerves, was estimated. These endings are probably caused by degenerative and/or regenerative changes.

Half the endings in the scleral conjunctiva of each eye were found in the supero-lateral quadrant, and the fewest endings in the infero-medial quadrant. This variation is probably related to the exposure of the sclera to the atmosphere.

The number of compact endings increases directly with age.

I am indebted to Dr. W. Ritchie Russell for permission to carry out this work on patients under his care, for his encouragement and valuable criticism. Help with the statistical data by Dr. E. H. Porter is gratefully acknowledged, as is the generous loan of apparatus by Dr. Graham Weddell.

\section{REFERENCES}

Frey, H. (1859). Histologie und Histochemie des Menschen. Engelmann, Leipzig.

Knüsel, O., and Vonwiller, P. (1922). Z. Augenheilk., 49, 157. Oppenheimer, D. R., Palmer, Elisabeth, and Weddell, G. (1958). J. Anat. (Lond.), 92, 321.

Ramón y Cajal, S. (1928). Degeneration and Regeneration of the Nervous System. trans. R. M. May. Oxford University Press, London.

Ruffini, A. (1906). Monit. zool. ital., 17, 16.

Speidel, C. C. (1941). Harvey Lect., 1940-1941, 36, 126.

Strughold, H., and Karbe, M. (1925). Z. Biol., 83 (n.s. 65), 297.

Weiss, P. (1941). In Third Symposium on Development and Growth. (Growth, 5 Suppl.) p. 163.

Zander, E., and Weddell, G. (1951). Brit. J. Ophthal., 35, 61 